\title{
Impact of Dose Algorithm and Calculation Angle Interval on in Vivo Dose Verification of Stereotactic Body Radiation Therapy: A Phantom Study
}

Shuxu Zhang ( $\sim$ gthzsx@163.com )

Affiliated Cancer Hospital \& Institute of Guangzhou Medical University https://orcid.org/0000-00019416-0782

\section{Songgui Luo}

Affiliated Cancer Hospital \& Institute of Guangzhou Medical University

Hui Yu

Affiliated Cancer Hospital \& Institute of Guangzhou Medical University

Shengqu Lin

Affiliated Cancer Hospital \& Institute of Guangzhou Medical University

Weibin Zhou

Affiliated Cancer Hospital \& institute of Guangzhou medicalUniversity

Guoquan Zhang

Affiliated Cancer Hospital \& Institute of Guangzhou Medical University

Quanbin Zhang

Affiliated Cancer Hospital \& Institute of Guangzhou Medical University

Pixiao Zhou

Affiliated Cancer Hospital \& Institute of Guangzhou Medical University

Ying Zhang

Affiliated Cancer Hospital \& Institute of Guangzhou Medical University

Yuliang Liao

Affiliated Cancer Hospital \& Institute of Guangzhou Medical University

Ruihao Wang

Affiliated Cancer Hospital \& Institute of Guangzhou Medical University

\section{Research}

Keywords: Stereotactic body radiation therapy, SRS MapCHECK, Exit dose, ET Verification Head Phantom, Dose calculation angle interval, In vivo dose

Posted Date: August 23rd, 2021

DOl: https://doi.org/10.21203/rs.3.rs-798868/v1 
License: (c) (i) This work is licensed under a Creative Commons Attribution 4.0 International License. Read Full License 


\section{Abstract}

\section{Objective}

To investigate the impact of different dose algorithms and calculation angle intervals (DCAI) on the in vivo dose (IVD) verification of small-field arc therapy in stereotactic body radiation therapy (SBRT).

\section{Methods}

We made an exit-dose-measuring and positioning device (EDPD) for the SRS MapCHECK (SMC) using polymethyl methacrylate (PMMA). Computed tomography data for the anthropomorphic head phantom, SMC, and EDPD combination were acquired with $1 \mathrm{~mm}$ slice thickness and spacing. SBRT partial arc plans were created using an SBRT cone, block, and a small square open field, with a gantry rotation angle of $60^{\circ}$. The dose distribution was calculated using three different dose algorithms [Pencil Beam (PB), CC Convolution (CCC), and Monte Carlo (MC)], with $1 \mathrm{~mm}$ isotropic resolution. We also used three different DCAls $\left(1^{\circ}, 3^{\circ}, 5^{\circ}\right)$ with the PB and CCC algorithms to calculate the dose distribution of each plan three times. The uncertainty of each control point for the MC algorithm was set to $1 \%$. The SMC was used to measure the exit dose outside the phantom for IVD verification, the detector plane was located $182.5 \mathrm{~mm}$ outside the scan center.

\section{Results}

Within the phantom, the minimum passing rate of 3D gamma analysis $(1 \% / 1 \mathrm{~mm})$ for the dose distributions calculated at different DCAls was $99.1 \%$, and the maximum relative deviation (RD) of the central point dose (CPD) was $<0.2 \%$. The average RD of the CPD for IVD verification was about $30 \%$ (range $16.71 \%-50.0 \%$ ) for $\mathrm{PB} ;-0.36 \% \pm 1.82 \%$ ( $1^{\circ} \mathrm{DCAl}$ ), $-3.18 \% \pm 7.83 \%\left(3^{\circ} \mathrm{DCAl}\right)$, and $3.69 \% \pm 11.56 \%$ $\left(1^{\circ} \mathrm{DCAl}\right)$ for $\mathrm{CCC}$; and $-0.38 \% \pm 0.76$ for the $\mathrm{MC}$ algorithm. The passing rates of $2 \mathrm{D}$ gamma analysis $(3 \% / 3 \mathrm{~mm})$ between the predicted exit dose and the IVD were $100 \%$ for MC and $>90 \%$ for the CCC algorithm at $1^{\circ} \mathrm{DCAl}$.

\section{Conclusion}

The DCAI for exit-dose calculations should be $\leq 1^{\circ}$ using the CCC algorithm. Furthermore, among the three algorithms verified in the current study, the $\mathrm{MC}$ algorithm showed the highest accuracy, followed by $\mathrm{CCC}$, with the PB algorithm having the worst performance. The PB algorithm is thus not suitable for exitdose calculation or IVD verification of SBRT.

\section{Introduction}

Stereotactic body radiation therapy (SBRT) has attracted increasing attention in line with recent developments in radiotherapy technology, due to its high positioning accuracy, fewer treatment fractions, and minor side effects. SBRT has accordingly been applied for the treatment of lung lesions ${ }^{[1-2]}$, brain metastases ${ }^{[3]}$, hepatocellular carcinoma ${ }^{[4]}$, pancreatic cancer ${ }^{[5]}$, and sacral and spinal metastases ${ }^{[6]}$. 
However, because SBRT delivers high doses of radiation in five fractions or less, any errors in delivery can be more pernicious than for conventional therapy. SBRT delivery thus requires high precision and accuracy, and benefits from patient-specific dose verification ${ }^{[7]}$. Because of the small dimensions and steep dose gradients of the beams used for SBRT, the AAPM Task Group 101 recommended that quality assurance tools for SBRT should have a minimum spatial resolution of approximately $1 \mathrm{~mm}{ }^{[8]}$.

The beam used for SBRT is usually a small cone with a diameter of $10-40 \mathrm{~mm}$, and arc radiotherapy is often used. The dose calculation for arc treatment is usually converted into the superposition of static irradiation fields with an equal gantry angle interval of $<5^{\circ}$ (arc increment for dose computation). However, the effects of different dose algorithms and calculation angle intervals (DCAls) (e.g. $1^{\circ}, 3^{\circ}$, and $5^{\circ}$ ) on dose verification, especially the calculation and verification of the exit dose (in vivo dose, IVD), has not been extensively explored for small-field arc irradiation in SBRT. In this study, we planned partial arc treatment with SBRT cone, block, and small square open field (SSOF), and compared the dose calculations using the Pencil Beam (PB) algorithm, CC conversion (CCC), and Monte Carlo (MC) algorithm. The dose distribution within the phantom and the exit dose outside the phantom were calculated with the different algorithms and angle intervals. The exit dose was measured using a high resolution two-dimensional (2D) diode detector array (stereotactic radiosurgery MapCHECK (SMC; Sun Nuclear Corp., Melbourne, FL, USA). The influences of the different algorithms and DCAls on the IVD verification of SBRT were investigated.

\section{Materials And Methods}

\subsection{Materials and equipment}

We used an Elekta Synergy clinical linear accelerator (Elekta, Crawley, UK) with 6-MV X-ray beams and a dose rate of $600 \mathrm{MU} / \mathrm{min}$. The 3DVH software system (V3.3.2), two-dimensional diode detector array SMC, and Patient V8.4 software system were from Sun Nuclear Corp. The 16-slice Big Bore computed tomography (CT) scanner was from Philips Medical Systems (Cleveland, OH, USA). The two treatment planning systems (TPS) were Pinnacle ${ }^{3}$ V9.10 (Radiation Oncology Systems, Fitchburg, WI, USA) with an SBRT cone calculation module, and Monaco V5.10 (Elekta). The anthropomorphic phantoms used in this study were an ET Verification Head Phantom (EVHP; The Phantom Laboratory, Salem, NY, USA) and the recently released StereoPHAN ${ }^{\top M}$ phantom (Sun Nuclear Corp). A set of special SBRT cones (diameters 10$40 \mathrm{~mm}$ ) were from TopSLANE Technology Limited (Shanghai, China). A homemade exit-dosemeasurement positioning device (EDPD) for SMC was made from polymethyl methacrylate $(8 \mathrm{~cm}$ wide, 2 $\mathrm{cm}$ thick), and was fixed on the couch top with a screw. The SMC can be held in the MapCheck Holder and its position can be adjusted accurately using the lifting knob (Fig. 1-a).

\subsection{CT image acquisition}

Similar to head and neck radiotherapy, a thermoplastic mask was used to fix the EVHP, and the SMC was held in the MapCheck Holder of the self-made EDPD. The distance from the front surface of the SMC to 
the scan center was $160 \mathrm{~mm}$, and the distance from the sagittal measuring plane to the scan center was $182.5 \mathrm{~mm}$. CT images of the SMC, EDPD, and EVHP combination were acquired with $1 \mathrm{~mm}$ slice thickness and spacing (Fig. 1-b).

\subsection{Treatment planning and delivery \\ 1.3.1 Cone arc treatment planning (Cone- ${ }_{\mathrm{PB}} 60^{\circ}$ )}

The CT images of the SMC and EVHP were transferred to the Pinnacle TPS. The center of the CT scan was used as an isocenter to create a $60^{\circ}$ arc treatment plan (Cone- ${ }^{-}$B $60^{\circ}$ ) for cones with different diameters $(10-40 \mathrm{~mm})$, in which the gantry rotated clockwise from $60^{\circ}$ to $120^{\circ}$, with a delivery dose of $300 \mathrm{MU}$ (Fig. 1-C). The PB algorithm for cone SBRT was used to calculate the dose distribution at DCAls of $1^{\circ}, 3^{\circ}$, and $5^{\circ}$, respectively, and the dose calculation grid was a $1 \mathrm{~mm}$ isotropic resolution. The SMC was included in the dose-calculation range to extract the planned exit-dose distribution. The finalized RT doses and RT plans were then exported to the SNC Patient 8.4 software system.

\subsubsection{Block arc treatment planning (Block-ccc 60\%)}

The CCC algorithm in Pinnacle V9.10 TPS cannot calculate the SBRT cone plan dose, and cone-shaped blocks (diameter 18-40 mm, height $85 \mathrm{~mm}$ ) were therefore copied from the SBRT cone (TopSLANE) using low-melting lead. After this, $60^{\circ}$ arc treatment plans (Block- ${ }^{-} \mathrm{CcC} 60^{\circ}$ ) for the different diameter blocks were created for the EVHP, and the dose distribution was calculated three times at DCAls of $1^{\circ}, 3^{\circ}$, and $5^{\circ}$, respectively, using the $\mathrm{CCC}$ algorithm. The isocenter, gantry rotation angle, delivery dose, calculation grid and range, and exportation of the plan files were the same as for Cone- ${ }_{\mathrm{PB}} 60^{\circ}$.

\subsubsection{SSOF arc treatment planning (SSOF-MC $60^{\circ}$ )}

The Monaco TPS (V 5.10) was used to create the SSOF arc treatment plan (SSOF- ${ }^{-}{ }_{\mathrm{MC}} 60^{\circ}$ ) for the EVHP. The dose was calculated using the MC algorithm. The uncertainty of each control point was set to $1 \%$, and the width of the SSOF was $18,22,26,30,36$, and $40 \mathrm{~mm}$, respectively. The isocenter, gantry rotation angle, delivery dose, calculation range, and exportation of the plan files were the same as for Cone- ${ }_{\mathrm{PB}} 60^{\circ}$.

\subsection{Dose measurement}

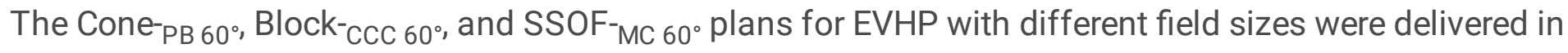
turn. The exit dose (in vivo) distributions outside the EVHP were measured using SMC and the self-made EDPD, and collected using SNC Patient 8.4 software.

\subsection{Data analysis}

\subsubsection{Dosimetric comparisons within EVHP (1D/3D)}

The central point dose (CPD) of the Cone- ${ }_{\mathrm{PB}} 60^{\circ}$ and Block- ${ }_{\mathrm{CCC}} 60^{\circ}$ plans for each cone/block calculated at $1^{\circ}, 3^{\circ}$, and $5^{\circ}$ intervals were extracted from the exported RT Dose and RT Plan using SNC Patient 8.4 
software and compared, and the relative deviation (RD) was calculated. A 3DVH software system was used to compare the differences among the $3 \mathrm{D}$ dose distributions calculated at $1^{\circ}, 3^{\circ}$, and $5^{\circ}$ DCAls, using volumetric gamma analysis (3D) with an absolute dose criterion of $1 \% / 1 \mathrm{~mm}$ (threshold value $10 \%)$

\subsubsection{IVD verification (1D/2D)}

The predicted exit-dose distributions of Cone- ${ }^{\mathrm{PB}} 60^{\circ}$, Block- ${ }^{-} \mathrm{CC} 60^{\circ}$, and SSOF- ${ }^{-} \mathrm{MC}_{6} 60^{\circ}$ at the measuring locations outside the EVHP were extracted using SNC Patient 8.4 software and compared with the IVD measured with SMC and the self-made EDPD. The comparison included the central point dose (1D), 2D gamma analysis with an absolute dose criterion of $3 \% / 3 \mathrm{~mm}$ (threshold value $20 \%$ ), and off-axis ratio.

\section{Results}

\subsection{Dosimetric comparisons within EVHP 2.1.1 Cone- ${ }^{-}$B $60^{\circ}$ plan}

The maximum RD among the CPDs of the Cone- ${ }_{\mathrm{PB}} 60^{\circ}$ plans calculated at different DCAls with the PB algorithm was $<0.1 \%$ for each cone. The minimum passing rate of $3 \mathrm{D}$ gamma analysis for dose distribution calculated at $1^{\circ}, 3^{\circ}$, and $5^{\circ}$ DCAls was $99.1 \%$ (Table 1 ). 
Table 1

Dosimetric comparison of Cone- $\mathrm{PB} 60^{\circ}$ plans calculated at $1^{\circ}, 3^{\circ}$, and $5^{\circ}$ DCAls using the PB algorithm

\begin{tabular}{|c|c|c|c|c|c|}
\hline \multirow[t]{2}{*}{ Field diameter (mm) } & \multicolumn{3}{|c|}{$\begin{array}{l}\text { 3D Gamma passing rate } \\
(1 \% / 1 \mathrm{~mm})\end{array}$} & \multicolumn{2}{|c|}{$\begin{array}{l}\text { Relative deviation } \\
(\Delta \%)\end{array}$} \\
\hline & $1^{\circ}$ vs. $3^{\circ}$ & $1^{\circ} \mathrm{vs} .5^{\circ}$ & $3^{\circ} \mathrm{vs} .5^{\circ}$ & $1^{\circ} \mathrm{vs} .3^{\circ}$ & $1^{\circ} \mathrm{vs} .5^{\circ}$ \\
\hline 40 & $100 \%$ & $100 \%$ & $100 \%$ & $0.02 \%$ & $-0.05 \%$ \\
\hline 35 & $100 \%$ & $99.9 \%$ & $100 \%$ & $0.02 \%$ & $-0.05 \%$ \\
\hline 30 & $100 \%$ & $100 \%$ & $100 \%$ & $0.02 \%$ & $-0.05 \%$ \\
\hline 26 & $100 \%$ & $99.9 \%$ & $100 \%$ & $0.02 \%$ & $-0.05 \%$ \\
\hline 22 & $100 \%$ & $99.8 \%$ & $99.9 \%$ & $0.01 \%$ & $-0.06 \%$ \\
\hline 18 & $100 \%$ & $99.6 \%$ & $99.7 \%$ & $0.01 \%$ & $-0.06 \%$ \\
\hline 14 & $100 \%$ & $99.6 \%$ & $99.7 \%$ & $0.02 \%$ & $-0.06 \%$ \\
\hline 10 & $100 \%$ & $99.1 \%$ & $99.2 \%$ & $0.02 \%$ & $-0.05 \%$ \\
\hline Average & $100 \%$ & $99.7 \%$ & $99.8 \%$ & $0.02 \%$ & $-0.05 \%$ \\
\hline SD & $0.00 \%$ & $0.30 \%$ & $0.28 \%$ & $0.00 \%$ & $0.01 \%$ \\
\hline
\end{tabular}

\subsubsection{Block-ccc $60^{\circ}$ plan}

The maximum RD between the CPDs calculated at different DCAls $\left(1^{\circ}\right.$ vs. $3^{\circ} ; 1^{\circ}$ vs. $\left.5^{\circ}\right)$ using the CCC algorithm was $<0.2 \%$. All passing rates were $100 \%$ for $3 \mathrm{D}$ gamma analysis performed among the dose distributions calculated at $1^{\circ}, 3^{\circ}$, and $5^{\circ}$ DCAls (Table 2 ). 
Table 2

Dosimetric comparison of Block- $\operatorname{ccc} 60^{\circ}$ plans calculated using the CCC algorithm at $1^{\circ}, 3^{\circ}$, and $5^{\circ}$ DCAls

\begin{tabular}{|c|c|c|c|c|c|}
\hline \multirow[t]{2}{*}{ Field diameter $(\mathrm{mm})$} & \multicolumn{3}{|c|}{ 3D Gamma passing rate $(1 \% / 1 \mathrm{~mm})$} & \multicolumn{2}{|c|}{ Relative deviation $(\Delta \%)$} \\
\hline & $1^{\circ}$ vs. $3^{\circ}$ & $1^{\circ}$ vs. $5^{\circ}$ & $3^{\circ}$ vs. $5^{\circ}$ & $1^{\circ}$ vs. $3^{\circ}$ & $1^{\circ}$ vs. $5^{\circ}$ \\
\hline 40 & $100 \%$ & $100 \%$ & $100 \%$ & $0.00 \%$ & $0.00 \%$ \\
\hline 35 & $100 \%$ & $100 \%$ & $100 \%$ & $0.19 \%$ & $0.15 \%$ \\
\hline 30 & $100 \%$ & $100 \%$ & $100 \%$ & $0.02 \%$ & $-0.02 \%$ \\
\hline 26 & $100 \%$ & $100 \%$ & $100 \%$ & $0.03 \%$ & $-0.01 \%$ \\
\hline 22 & $100 \%$ & $100 \%$ & $100 \%$ & $0.14 \%$ & $0.05 \%$ \\
\hline 18 & $100 \%$ & $100 \%$ & $100 \%$ & $0.01 \%$ & $-0.04 \%$ \\
\hline
\end{tabular}

\subsection{IVD verification of EVHP}

\subsubsection{Features of exit-dose distribution}

In this study, the measuring plane of the exit dose was located $182.5 \mathrm{~mm}$ from the isocenter. When the DCAI was $1^{\circ}$, the irradiating/projecting range of cone beams with adjacent gantry angles overlapped, and the predicted exit-dose distributions were therefore continuous for each cone beam (Fig. 2). However, a poorer continuity of dose distribution was associated with a smaller cone diameter and larger calculation interval angle, resulting in a completely independent circular or elliptical dose distribution area (Fig. 2).

\subsubsection{IVD verification using PB algorithm}

There were significant differences between the predicted CPDs of Cone- ${ }^{\mathrm{PB}} 60^{\circ}$ plans and the measured exit dose at the sagittal plane (Table 3). The minimum RD was $>16 \%$, the maximum was $50 \%$, and the average was about $30 \%$. Furthermore, when the exit-dose distributions were calculated using the PB algorithm at DCAls of $3^{\circ}$ and $5^{\circ}$, the planned off-axis ratios of exit doses at the central axis resembled a sine curve and were not consistent with the measurements (Fig. 3). A greater variation in amplitude of the planned off-axis ratios was associated with a smaller cone diameter and a larger DCAl (e.g. $10 \mathrm{~mm}$ diameter cone and $\left.5^{\circ} \mathrm{DCAl}\right)$. 
Table 3

Dosimetric comparison between predicted central point doses of Cone- ${ }^{\mathrm{PB}} 60^{\circ}$ and IVD measured at $182.5 \mathrm{~mm}$ outside the EVHP

\begin{tabular}{|llll|}
\hline Cone diameter $(\mathrm{mm})$ & \multicolumn{3}{c|}{ Relative deviation $(\boldsymbol{\Delta} \%)$} \\
\cline { 2 - 4 } & $\mathbf{1}^{\circ}$ & $\mathbf{3}^{\circ}$ & $\mathbf{5}^{\circ}$ \\
\hline 40 & $30.38 \%$ & $29.23 \%$ & $29.02 \%$ \\
\hline 35 & $31.61 \%$ & $24.74 \%$ & $18.47 \%$ \\
\hline 30 & $32.39 \%$ & $36.90 \%$ & $16.71 \%$ \\
\hline 26 & $33.51 \%$ & $34.69 \%$ & $43.52 \%$ \\
\hline 18 & $33.60 \%$ & $25.18 \%$ & $50.00 \%$ \\
\hline 14 & $32.70 \%$ & $26.32 \%$ & $38.09 \%$ \\
\hline Average & $25.75 \%$ & $36.41 \%$ & $20.47 \%$ \\
\hline SD & $22.53 \%$ & $26.31 \%$ & $22.44 \%$ \\
\hline
\end{tabular}

\subsubsection{IVD verification using CCC algorithm}

The dosimetric comparison between the predicted CPDs of Block-CCC $60^{\circ}$ plans and measurements of the exit dose at the sagittal plane are shown in Table 4. When the DCAl was low (e.g. $1^{\circ}$ ), the maximum $\mathrm{RD}$ between the measurements and calculations was $<3 \%$, but the maximum RD was nearer $20.0 \%$ when the angle interval was increased to $5^{\circ}$. The passing rates of 2D gamma analysis $(3 \% / 3 \mathrm{~mm})$ between the SMC measurements and the predicted exit dose at different DCAls are shown in Table 4. The average passing rates for DCAls of $1^{\circ}, 3^{\circ}$, and $5^{\circ}$ were $99.63 \% \pm 0.55 \%, 90.32 \% \pm 14.22 \%$, and $87.27 \% \pm 13.45 \%$, respectively.

The CCC algorithm planned off-axis ratios and measurements on the centerline of the exit-dose measurement plane are shown in Fig. 4. A more pronounced variation in the off-axis ratio was associated with a smaller blocking aperture and a larger calculation angle interval. When the blocking aperture was increased and the DCAI was decreased, the difference between the calculated off-axis ratios and the measurement was less evident and eventually became coincident (e.g. when the blocking aperture was $40 \mathrm{~mm}$ and the DCAI was $3^{\circ}$ ). 
Table 4

Dosimetric comparison between predicted doses of Block- ${ }^{-C c C} 60^{\circ}$ and IVD measured at $182.5 \mathrm{~mm}$ outside the EVHP

\begin{tabular}{|c|c|c|c|c|c|c|}
\hline \multirow{2}{*}{$\begin{array}{l}\text { Cylinder } \\
\text { diameter } \\
(\mathrm{mm})\end{array}$} & \multicolumn{2}{|l|}{$5^{\circ} \mathrm{DCAI}$} & \multicolumn{2}{|l|}{$3^{\circ} \mathrm{DCAl}$} & \multicolumn{2}{|l|}{$1^{\circ} \mathrm{DCAl}$} \\
\hline & $\begin{array}{l}\text { Relative } \\
\text { deviation } \\
(\Delta \%)\end{array}$ & $\begin{array}{l}\text { Gamma } \\
\text { passing } \\
\text { rate }\end{array}$ & $\begin{array}{l}\text { Relative } \\
\text { deviation } \\
(\Delta \%)\end{array}$ & $\begin{array}{l}\text { Gamma } \\
\text { passing } \\
\text { rate }\end{array}$ & $\begin{array}{l}\text { Relative } \\
\text { deviation } \\
(\Delta \%)\end{array}$ & $\begin{array}{l}\text { Gamma } \\
\text { passing } \\
\text { rate }\end{array}$ \\
\hline 40 & $2.87 \%$ & $97.9 \%$ & $1.73 \%$ & $100 \%$ & $-0.69 \%$ & $100.0 \%$ \\
\hline 35 & $-7.46 \%$ & $61.8 \%$ & $-5.30 \%$ & $64.4 \%$ & $0.75 \%$ & $100.0 \%$ \\
\hline 30 & $-2.95 \%$ & $95.8 \%$ & $4.39 \%$ & $99.0 \%$ & $2.45 \%$ & $98.6 \%$ \\
\hline 26 & $-5.75 \%$ & $83.3 \%$ & $1.28 \%$ & $96.0 \%$ & $-0.90 \%$ & $99.5 \%$ \\
\hline 22 & $20.66 \%$ & $91.7 \%$ & $-3.85 \%$ & $99.5 \%$ & $-0.76 \%$ & $100.0 \%$ \\
\hline 18 & $14.75 \%$ & $93.1 \%$ & $-17.33 \%$ & $83.0 \%$ & $-2.98 \%$ & $99.7 \%$ \\
\hline Average & $3.69 \%$ & $87.27 \%$ & $-3.18 \%$ & $90.32 \%$ & $-0.36 \%$ & $99.63 \%$ \\
\hline SD & $11.56 \%$ & $13.45 \%$ & $7.83 \%$ & $14.22 \%$ & $1.82 \%$ & $0.55 \%$ \\
\hline
\end{tabular}

\subsubsection{IVD verification using the $M C$ algorithm}

The maximum RD between the CPDs calculated using the MC algorithm and the measurements at the sagittal plane $182.5 \mathrm{~mm}$ from the isocenter was $<1.5 \%$. The average RD was $-0.38 \% \pm 0.76 \%$. The $2 \mathrm{D}$ gamma passing rate $(2 \% / 2 \mathrm{~mm})$ of the predicted dose and the in vivo measurement was $>98.0 \%$, and the gamma passing rate was $100.0 \%(3 \% / 3 \mathrm{~mm})$ (Table 5$)$. 
Table 5

Dosimetric comparison between predicted dose of SSOF- ${ }_{\mathrm{MC}} 60^{\circ}$ plan and IVD measured at $182.5 \mathrm{~mm}$ outside the EVHP

\begin{tabular}{|c|c|c|c|c|}
\hline \multirow[t]{2}{*}{ Square field (mm) } & \multicolumn{2}{|c|}{ 2D gamma passing rate } & \multirow{2}{*}{$\begin{array}{l}\text { Dose Difference } \\
\text { (cGy) }\end{array}$} & \multirow{2}{*}{$\begin{array}{l}\text { Relative Deviation } \\
(\Delta \%)\end{array}$} \\
\hline & $2 \% / 2 \mathrm{~mm}$ & $3 \% / 3 \mathrm{~mm}$ & & \\
\hline $40 \times 40$ & $99.3 \%$ & $100 \%$ & 0.10 & $-0.35 \%$ \\
\hline $36 \times 36$ & $98.8 \%$ & $100 \%$ & 0.05 & $0.21 \%$ \\
\hline $30 \times 30$ & $98.7 \%$ & $100 \%$ & -0.12 & $-0.53 \%$ \\
\hline $26 \times 26$ & $99.2 \%$ & $100 \%$ & -0.20 & $-1.06 \%$ \\
\hline $22 \times 22$ & $98.4 \%$ & $100 \%$ & -0.10 & $-0.65 \%$ \\
\hline $18 \times 18$ & $98.7 \%$ & $100 \%$ & -0.20 & $-1.48 \%$ \\
\hline $14 \times 14$ & $98.4 \%$ & $100 \%$ & -0.02 & $-0.19 \%$ \\
\hline $10 \times 10$ & $99.6 \%$ & $100 \%$ & 0.08 & $1.01 \%$ \\
\hline Average & $98.89 \%$ & $100.00 \%$ & -0.05 & $-0.38 \%$ \\
\hline SD & $0.44 \%$ & $0.00 \%$ & 0.11 & $0.76 \%$ \\
\hline
\end{tabular}

\section{Discussion}

For the Cone- ${ }^{-}{ }_{\mathrm{PB}} 60^{\circ}$ plan of the EVHP with a larger DCAl (such as $3^{\circ}$ or $5^{\circ}$ ), the adjacent beams showed little overlaps at the sagittal measuring plane of the exit dose, and an independent circular or elliptical dose distribution area was predicted. In the meantime, the planned off-axis ratio at the central axis resembled a sine curve and was much lower than the measured. This is mainly because the Pinnacle ${ }^{3}$ SBRT dose algorithm (PB) computes the dose to the target based on interpolation of the absorbed dose as measured in water, without density correction. In the current study, the air gap between the phantom surface and SMC was about $8-10 \mathrm{~cm}$, and this was taken as the equivalent tissue when the SBRT PB algorithm was adopted to calculate the exit-dose distribution. The calculated equivalent depth of beam penetration was thus far more significant than the actual depth, leading to a large deviation between the planned and measured results. The minimum RD of the CPD for Cone- ${ }^{\mathrm{PB}} 60^{\circ}$ plans was $>16 \%$, with a maximum of $50 \%$ and average of about $30 \%$. The PB algorithm is therefore not suitable for calculating the exit dose and cannot be used with IVD verification.

The most commonly employed dose algorithms in TPS are the Acuros XB (AXB), analytical anisotropic algorithm (AAA), CCC, $P B$, and $M C$. The $C C C$ and $P B$ algorithms were usually used in early TPS, while the $A A A$ and $A X B$ algorithms are used in current Eclipse systems ${ }^{[9]}$. A previous report showed that the 
calculation results of the AXB and AAA algorithms were largely consistent with the MC simulation results in uniform media, while the difference between the MC simulation and AAA algorithm was more significant than that for the AXB algorithm in heterogeneous media ${ }^{[10]}$. Similarly, another study [11] compared and analyzed the dose-calculation accuracy of the AAA and AXB algorithms in lung SBRT planning, and found a larger systematic dose error with the AAA compared with the AXB algorithm. Another previous study ${ }^{[12]}$ indicated that, although the AAA and AXB calculations differed in terms of the D95, Dmax, TV95, and HI of PTV in patients with left lung cancer, the magnitude of the differences was too small to have any clinically observable effect. Alghamdi et al. ${ }^{[13]}$ compared the dose calculation accuracies of the AXB, AAA, and PB algorithms in four different-density media, and found that the AXB algorithm results were closest to the actual measured dose.

In the current study, the exit dose could be predicted precisely when the CCC algorithm was used to calculate the dose distribution of arc therapy in SBRT with a DCAI of $1^{\circ}$, with a maximum RD between the measured and calculated results of $<3 \%$. The planned off-axis ratio curve on the central axis was consistent with the measurements, and its difference was minimal. The average passing rate of 2D gamma analysis $(3 \% / 3 \mathrm{~mm})$ between the predicted exit doses and the in vivo measurements was $99.63 \%$ $\pm 0.55 \%$, indicating that the CCC algorithm and $1^{\circ} \mathrm{DCAl}$ were suitable for calculating the exit dose and IVD verification.

Most routine radiotherapy plans are currently verified using the pretreatment dose in a homogeneous phantom. However, this can only detect the difference between the calculated and measured doses in the phantom, and fails to detect differences between the planned dose and the dose actually received by the patient. Some studies have shown minimal significance of pretreatment verification with a homogeneous phantom. However, even if the calculated results are consistent with the measurements in the phantom, this cannot guarantee that the dose received by the patient is consistent with the planned dose ${ }^{[14]}$. Many factors might affect the dose received by the patient, including setup error, body changes, gastrointestinal filling, respiratory movements, and the stability of the radiotherapy equipment. The true accuracy of the delivered dose can only be reflected by real-time online measurement of the received dose in the patient's body during radiotherapy, or by measuring the exit dose outside the patient.

Mijnheer et al. ${ }^{[15]}$ suggested that online IVD measurements should be conducted for patients receiving radical radiotherapy, to detect dose errors caused by various factors in the overall treatment procedure. Pretreatment dose verification alone fails to detect $>50 \%$ of severe dose errors during the actual therapy process. Furthermore, in vivo measurements will identify potential errors in dose calculation, data transfer, patient setup, dose delivery, and changes in patient anatomy, suggesting that all treatments with curative intent should be verified through IVD measurements combined with pretreatment checks ${ }^{[16]}$. Another study reported that IVD monitoring detected $>74 \%$ of errors caused by equipment faults or human error, suggesting that real-time IVD verification should be used for first radiotherapy treatments [17]. 
Compared with pretreatment dose verification with a homogeneous phantom, IVD verification can evaluate the results of plan implementation more directly and accurately. The main IVD verification methods currently include the following: (1) point dosimetry, including semiconductor detectors, optically stimulated luminescent dosimeters, thermoluminescence dosimeters (TLD), metal-oxide-semiconductor field-effect transistors (MOSFET), and plastic scintillation detectors; (2) transmission dose detection methods, such as Delta4 Discover (transmission type) and integrated quality control monitors, with the main advantages of high resolution, large-size field measurement, non-coplanar irradiation measurement, and real-time measurement during treatment, without affecting the treatment process; (3) log file analysis; and (4) EPID real-time monitoring ${ }^{[18]}$. In contrast, the main disadvantages of these IVD methods include lower spatial differentiation rate, fewer measurement points, and poor positioning accuracy of point dosimetry; lack of sensitivity to setup error and intrafraction motion for EPID; only detecting the incoming dose and lack of sensitivity to errors caused by patient body changes, respiratory movement and setup error for transmission dose detection; and log file analysis is not an independent measurement method. At least two monitoring methods are thus recommended ${ }^{[19]}$. In the present study, we only explored the impacts of three dose algorithms and DCAls on IVD verification of small field partial arc SBRT with a phantom study. We aim to investigate full arc treatment of small fields, volumetric modulated arc therapy and intensity-modulated radiation therapy, and more algorithms in subsequent research. We will then carry out IVD verification of actual patients based on the phantom study.

The commonly used 2D and 3D dose verification systems, such as MatriXX, ArcCHECK, Delta4, and MapCHECK, cannot perform direct real-time IVD verification. Based on SMC and a self-made EDPD, we therefore investigated the impact of different dose algorithms and DCAls on IVD verification of SBRT. The results indicated that, for IVD verification, the DCAI of SBRT arc therapy should be $1^{\circ}$ when the CCC algorithm was used. In addition, the MC algorithm was the best of the three tested algorithms, followed by the CCC algorithm, both of which could calculate the exit dose accurately, while the PB algorithm was the worst and was not suitable for exit-dose calculation or IVD verification. SMC can thus be used to conduct IVD verification of SBRT partial arc therapy.

\section{Abbreviations}

Dose Calculation Angle lintervals (DCAl)

in vivo Dose (IVD)

Stereotactic Body Radiation Therapy (SBRT)

Exit-Dose-measuring and Positioning Device (EDPD)

SRS MapCHECK (SMC)

Pencil Beam (PB) 
CC Convolution (CCC)

Monte Carlo (MC)

Relative Deviation (RD)

Central Point Dose (CPD)

Small Square Open Field (SSOF)

Treatment Planning Systems (TPS)

ET Verification Head Phantom (EVHP)

Acuros XB (AXB)

Analytical Anisotropic Algorithm (AAA)

Electronic Portal Imaging Device (EPID )

\section{Declarations}

\section{- Ethical Approval and Consent to participate}

This study was approved by the Ethics Committee of the Affiliated Cancer Hospital \& Institute of Guangzhou Medical University. Individual informed consent was waived as this was a phantom study.

\section{- Consent for publication}

Not applicable

\section{- Availability of supporting data}

Not applicable

\section{- Competing interests}

The authors declare that they have no competing interests.

\section{- Funding}

Not applicable 


\section{- Authors' contributions}

SZ designed the study, analyzed the data, and wrote the manuscript. SL and HY completed the treatment planning and data collection. SZ and SL wrote the manuscript. GZ, QZ, YL, and RW evaluated the treatment plan. WZ, PZ, and $Y Z$ completed the patient selection and data collection.

\section{- Acknowledgements}

This work was supported financially by Guangdong Provincial Natural Science Fund (Grant No. 2021A1515011329), Guangzhou science and technology project (Grant No. 201804010297).

\section{References}

1. Pokhrel D, Halfman M, Sanford L. FFF-VMAT for SBRT of lung lesions: Improves dose coverage at tumor-lung interface compared to flattened beams. J Appl Clin Med Phys.2019 Dec 20.

2. Fernandez C, Grills IS, Ye H, et al. Stereotactic image-guided lung radiotherapy (SBRT) for clinical early-stage NSCLC: A long-term report from a multi-institutional database of patients treated with or without a pathologic diagnosis. Pract Radiat Oncol. 2019 Dec 11.

3. Kumar AMS, Miller J, Hoffer SA, et al. Postoperative hypofractionated stereotactic brain radiation (HSRT) for resected brain metastases: improved local control with higher BED10. J Neurooncol.2018 Sep; 139(2):449-454.

4. Rusu I, Thomas TO, Roeske JC, et al. Failure mode and effects analysis of linac-based liver stereotactic body radiotherapy. Med Phys.2019 Dec 14.

5. Arcelli A, Guido A, Buwenge $M$, et al. Higher biologically effective dose predicts survival in SBRT of pancreatic cancer: A multicentric analysis (PAULA-1). Anticancer Res.2020 Jan;40(1):465-472.

6. Dunne EM, Sahgal A, Lo SS, et al. International consensus recommendations for target volume delineation specific to sacral metastases and spinal stereotactic body radiation therapy (SBRT). Radiother Oncol. 2019 Dec 21; 145:21-29.

7. Sarkar V, Huang L, Huang YJ, et al. Head to head comparison of two commercial phantoms used for SRS QA. J Radiosurg SBRT. 2016; 4(3):213-223.

8. Benedict SH, Yenice KM, Followill D, et al. Stereotactic body radiation therapy: The report of AAPM Task Group 101. Med Phys. 2010 Aug; 37(8):4078-101.

9. Fogliata A, Nicolini G, Clivio A, Vanetti E, Cozzi L. Accuracy of Acuros XB and AAA dose calculation for small fields with reference to RapidArc( $\left({ }^{\circledR}\right)$ stereotactic treatments. Med Phys. 2011 Nov; 38(11): 6228-37.

10. Krishna GS, Srinivas V, Reddy PY. Clinical implications of Eclipse analytical anisotropic algorithm and Acuros XB algorithm for the treatment of lung cancer. J Med Phys. 2016 Oct-Dec;41(4):219-223. 
11. Stathakis S, Esquivel C, Quino $L$ V, et al. accuracy of the small field dosimetry using the acuros XB

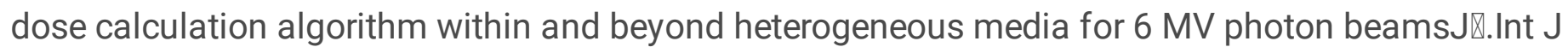
Med Phys Clin Eng Radiat Oncol, 2012, 3(1): 78-87.

12. Kawai D, Takahashi R, Kamima T, Baba H, Yamamoto T, Kubo Y, Ishibashi S, Higuchi Y, Tani K, Mizuno N, Jinno S, Tachibana H. Variation of the prescription dose using the analytical anisotropic algorithm in lung stereotactic body radiation therapy. Phys Med. 2017 Jun;38:98-104.

13. Alghamdi S, Tajaldeen A. Evaluation of dose calculation algorithms using different density materials for in-field and out-of-field conditions. Exp Oncol. 2019 Mar;41(1):46-52.

14. Zhen $H$, Nelms BE, Tome WA, et al. Moving from gamma passing rates to patient DVH-based QA metrics in pre-treatment dose QA. Med Phys. 2011 Oct;38(10):5477-89.

15. Mijnheer B, Beddar S, Izewska J, et al. In vivo dosimetry in external beam radiotherapy[J]. Med Phys, 2013, 40(7): 070903.

16. Mans A, Wendling M, McDermott LN, et al. Catching errors with in vivo EPID dosimetry[J]. Med Phys, 2010, 37(6):2638-2644.

17. Bojechko C, Phillps M, Kalet A, et al. A quantification of the effectiveness of EPID dosimetry and software-based plan verification systems in detecting incidents in radiotherapy[J]. Med Phys, 2015, 42(9): 5363-9.

18. McCurdy BMC, McCowan PM. In vivo dosimetry for lung radiotherapy including SBRT. Phys Med. 2017 Dec;44:123-130.

19. Esposito M, Villaggi E, Bresciani S, Cilla S, Falco MD, Garibaldi C, Russo S, Talamonti C, Stasi M, Mancosu P. Estimating dose delivery accuracy in stereotactic body radiation therapy: A review of invivo measurement methods. Radiother Oncol. 2020 Aug;149:158-167.

\section{Figures}




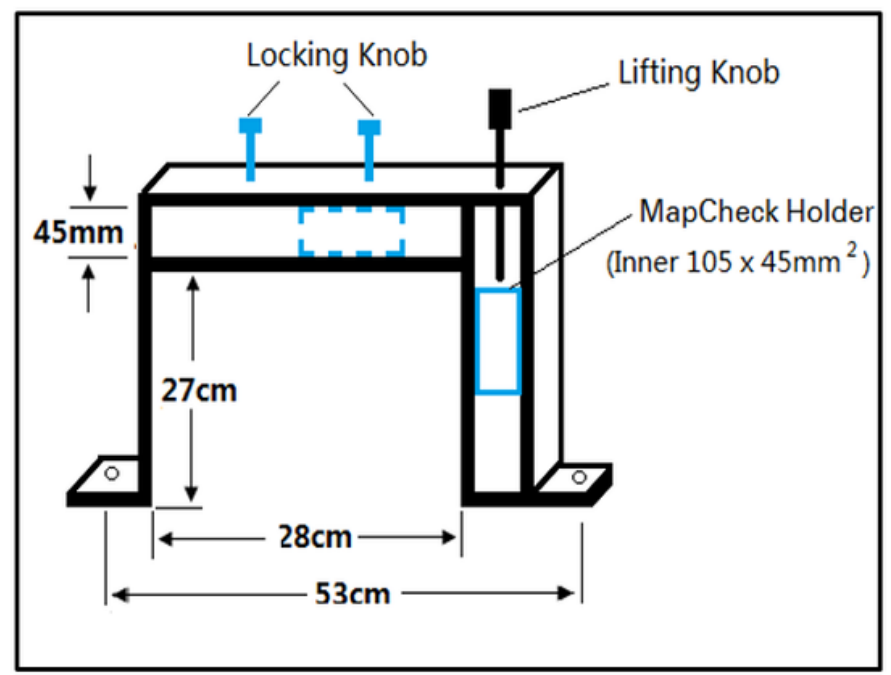

a. Schematic diagram of homemade EDPD.

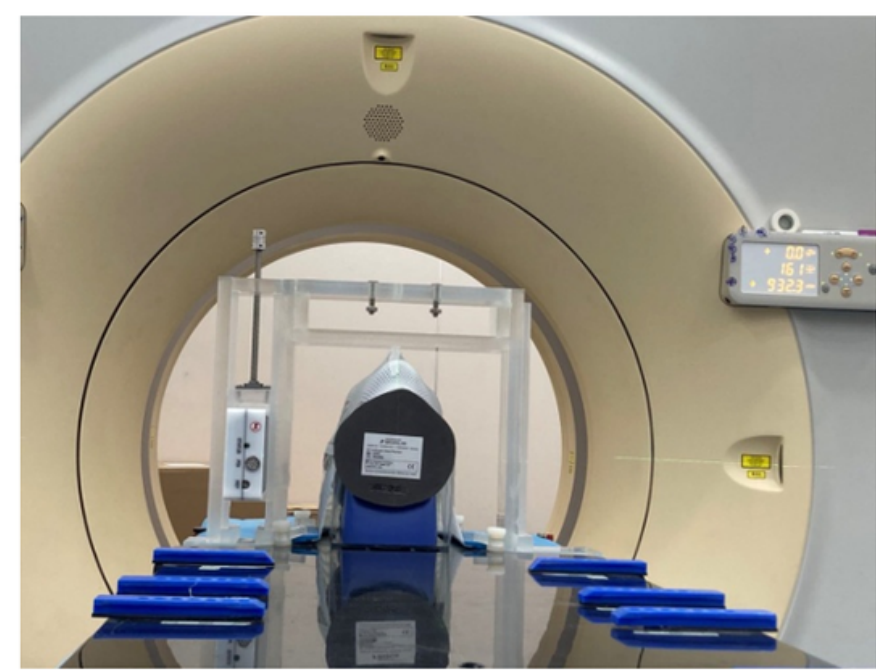

b. Acquiring CT image of SMC and EVHP.

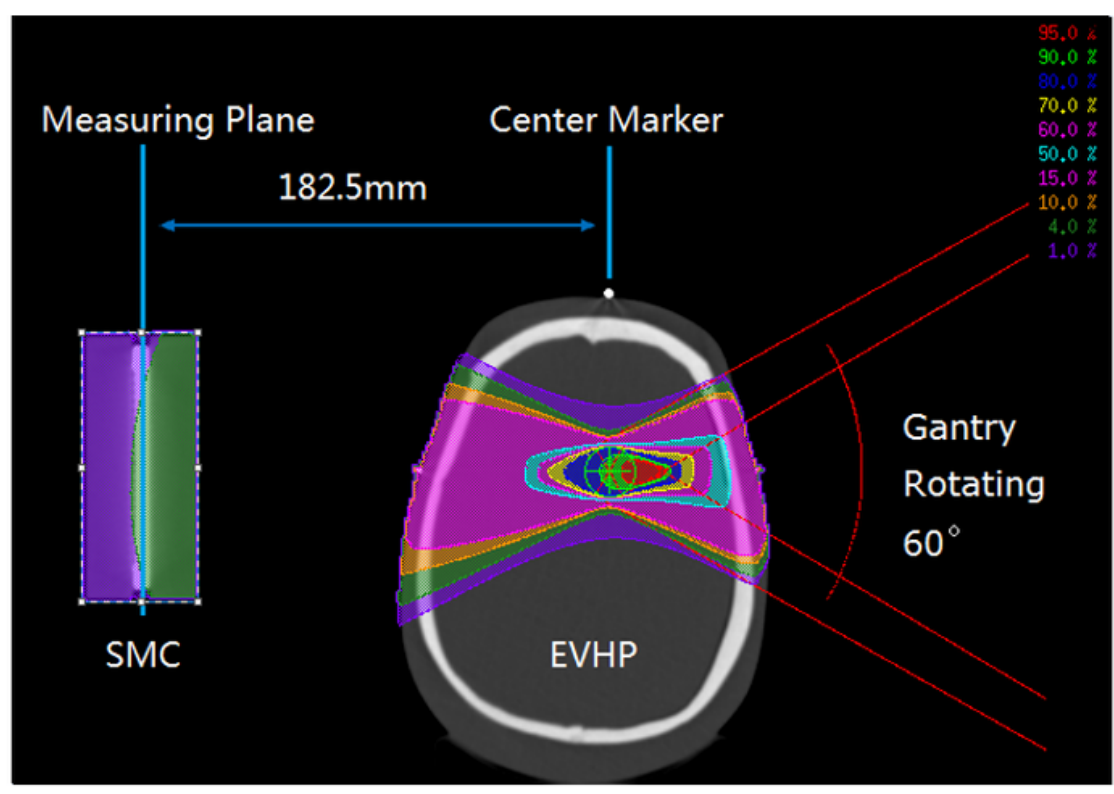

c. Planning cone-PB $60^{\circ}$ plan with SMC and EVHP.

\section{Figure 1}

Diagram of EDPD, CT imaging, treatment planning, and exit-dose measurement. 

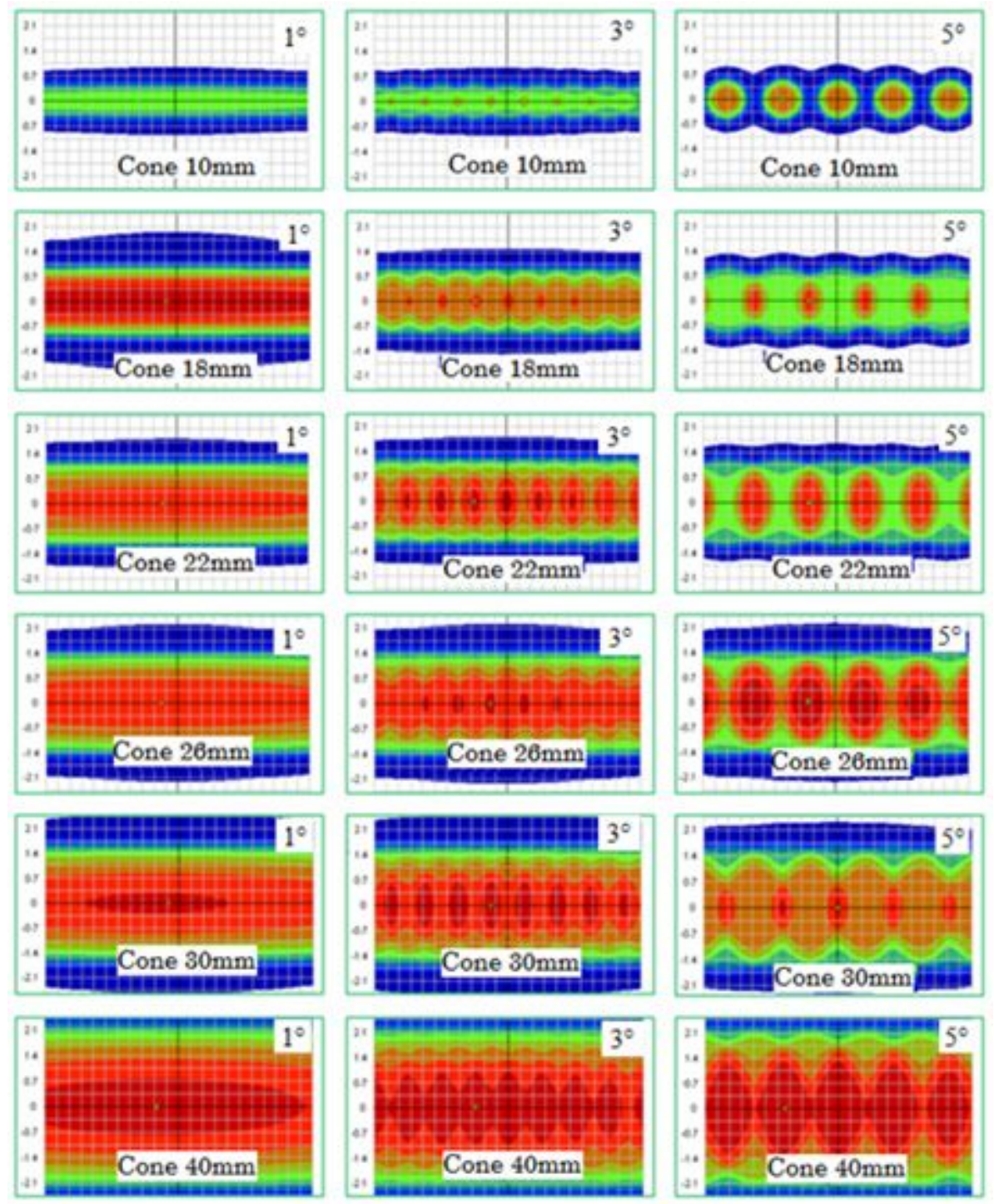

Figure 2

Characteristics of exit-dose distributions predicted using the PB algorithm at $1^{\circ}, 3^{\circ}$, and $5^{\circ}$ DCAls. 


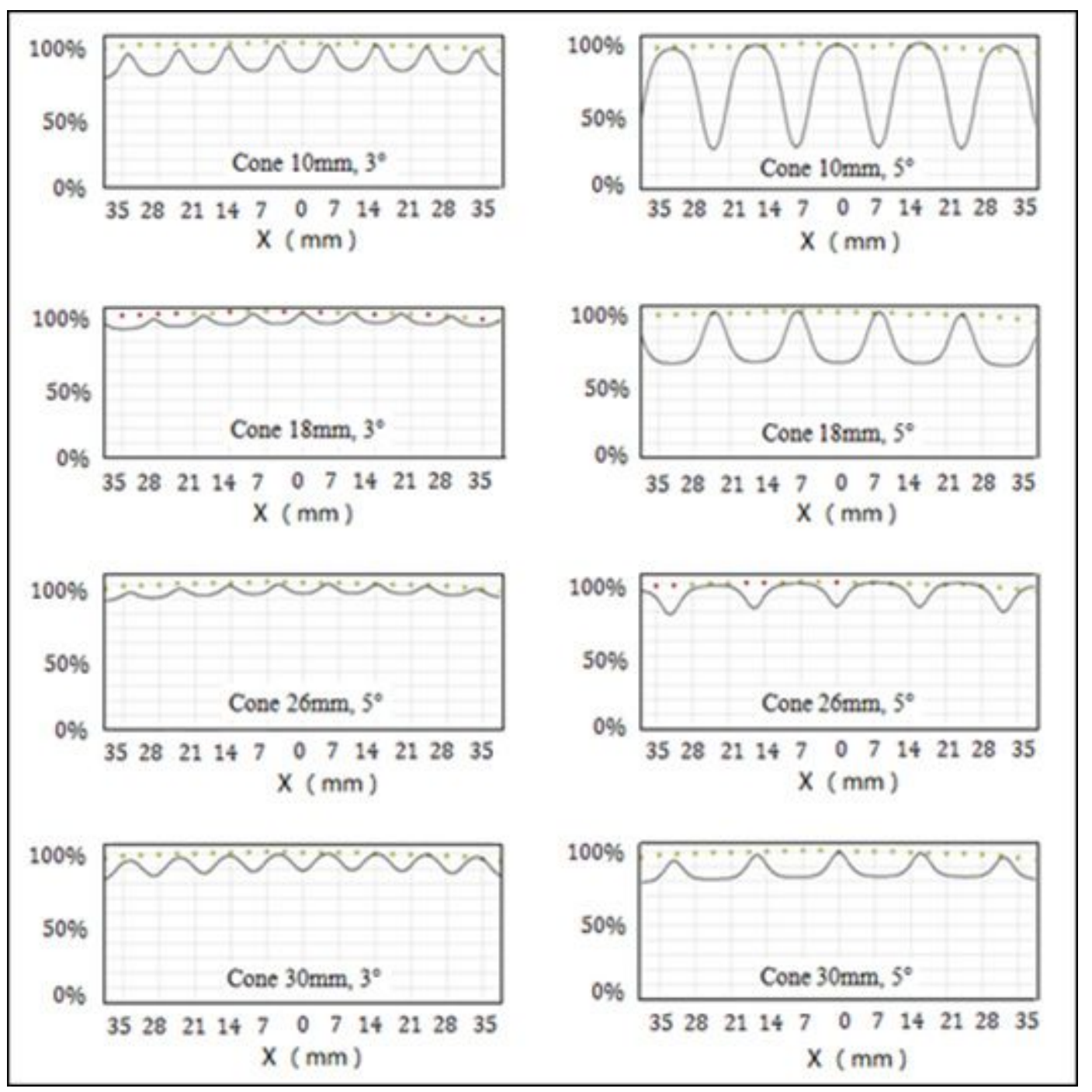

Figure 3

Off-axis ratios (solid line) of Cone-PB $60^{\circ}$ predicted by the PB algorithm on the central axis and measurements (dots). 


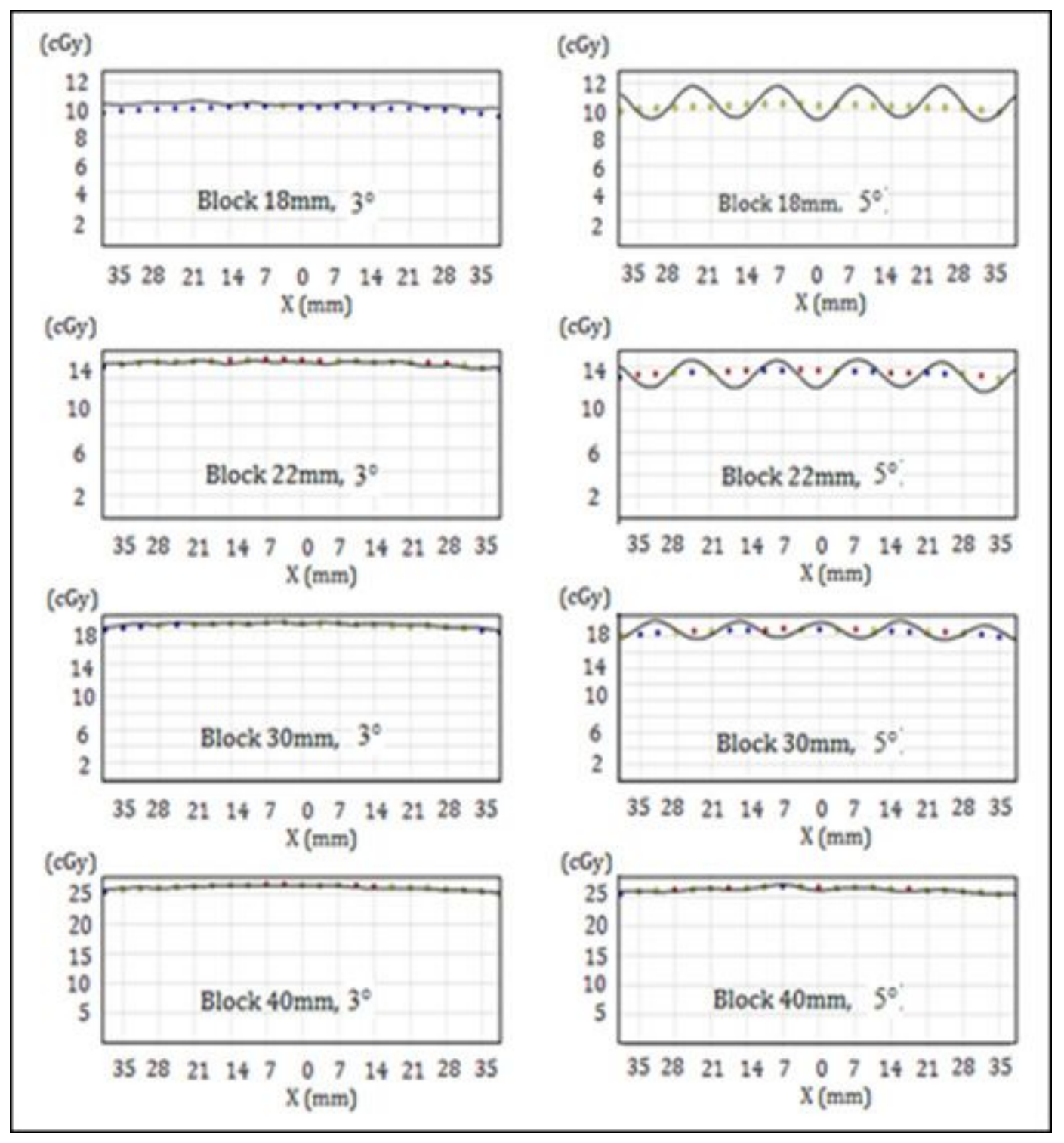

\section{Figure 4}

CCC algorithm predicted off-axis ratio (solid line) of Block-CCC $60^{\circ}$ and in vivo-measured off-axis ratio (dots). 